\title{
Expansion of directly observed treatment, short- course in urban and public-private mix areas of Bangladesh
}

This article was published in the following Dove Press journal:

Research and Reports in Tropical Medicine

I December 2010

Number of times this article has been viewed

\author{
Mohiuddin Hussain Khan ${ }^{1,2}$ \\ ABM Tauhidul Islam ${ }^{3}$ \\ 'Department of Drug Management \\ and Policy, Institute of Medical, \\ Pharmaceutical and Health Sciences, \\ Kanazawa University, Kanazawa City, \\ Ishikawa, Japan; ${ }^{2}$ World Health \\ Organization, Dhaka, Bangladesh; \\ 'World Health Organization, Geneva, \\ Switzerland
}

\begin{abstract}
Success with tuberculosis (TB) control was relatively poor in urban centers and some of the difficult to reach areas (eg, workplaces and prisons) in Bangladesh prior to 2004. This may be due to the complexities that exist within urban health care systems and the unique health care-seeking behaviors of urban citizens. A variety of health care institutions and providers are providing health services in urban areas. To increase the case detection of TB suspects, a number of initiatives were undertaken by the National Tuberculosis Control Program. The total geographic area was divided among different nongovernmental organizations to implement TB care services. Medical academic institutions and hospitals, industrial workplaces, and prisons were involved to provide DOTS (directly observed treatment, shortcourse, as an internationally recommended strategy for TB control) services. As a result, an increased number of TB cases were identified and reported in the urban and public-private mix areas. The TB case detection rate in the six city corporation areas increased from $60 \%$ in 2005 to $74 \%$ in June 2007, with an $87 \%$ sputum conversion rate in 2006 and an $84.5 \%$ treatment success rate in 2005. However, decreased treatment success rates were observed in hospital settings and in prisons, which might be due to ineffective referrals provided upon discharge. It is crucial to engage all care providers to increase DOTS coverage and to control TB in urban and special settings.
\end{abstract}

Keywords: public health, tuberculosis, developing country, program, expansion, DOTS, public-private mix

\section{Introduction}

Urban tuberculosis (TB) control has always been a challenge for national TB control programmes in many parts of the world, and Bangladesh is no exception. ${ }^{1-3}$ Bangladesh ranks sixth among the 22 high-burden TB countries in the world. ${ }^{1,2}$ The National TB Control Program adopted directly observed treatment, short-course (DOTS, an internationally-recommended strategy for TB control) at the beginning of $1993 .{ }^{3}$ However, the program did not gain momentum until 2004. ${ }^{4,5}$ To boost the activities of the National Tuberculosis Control Program, the entire country had been assigned by geographic area to several nongovernmental organizations (NGOs) during 2003 and $2004{ }^{3,6}$ Gradually, after expansion and further strengthening of the government-NGO collaboration, the performance of the TB control program increased significantly throughout the entire country. ${ }^{7}$ Nevertheless, performance continued to be poor in these urban areas compared with the trends observed in the rural areas. ${ }^{7}$ Access and characteristics of health services, health-seeking behaviors of urban dwellers, and many other social factors contributed to these challenges. ${ }^{8,9}$ To overcome the challenges
Correspondence: Mohiuddin Hussain Khan Department of Drug Management and Policy, Institute of Medical, Pharmaceutical and Health Sciences, Kanazawa University, Kakumamachi, Kanazawa City, Ishikawa, Japan $920-1192$

Tel +88017 II 5674 I

Fax +81762344402

Email mohiuddin_khn@yahoo.com 
and to reach the TB control objectives, several new control strategy initiatives were undertaken. ${ }^{10}$

\section{DOTS in city corporation areas}

Utilization of health care services and health-seeking behaviors of TB patients varies significantly across the city corporation areas of Bangladesh. ${ }^{11}$ Beginning in 2004, different areas (wards) of the six city corporations had been assigned to a number of collaborative partner NGOs to provide DOTS services through their existing urban health care facilities. ${ }^{6,7}$ These NGOs cover an estimated population of 14.3 million through 299 directly observed treatment (DOT) centers in the city corporation areas, along with additional government health facilities. ${ }^{12}$ Eighty-three of these treatment centers were additionally equipped with sputum microscopy facilities.

NGO health centers provide TB services as a part of their comprehensive primary health care service package, and are assigned by the respective city corporations for specific catchment areas and population coverage (100,000-300,000 residents). ${ }^{5}$ Typically, these centers are staffed with 1-3 medical officer(s), 2-4 paramedics, 1-2 laboratory technician(s), and 2-3 support staff, depending on their customer volume and population coverage. ${ }^{13}$ Before the initiation of DOTS services, these health centers were provided with the necessary logistic support free of cost from the National Tuberculosis Control Program after signing agreements. Some of the DOTS centers provide a full range of DOTS services, including sputum microscopy facilities, whereas other centers provide identification and screening of TB suspects, collection of sputum specimens, and provision of DOT, essentially functioning as referral centers without microscopy facilities. ${ }^{14}$

To strengthen TB control services in the city corporation areas, technical staff members were assigned to coordinate the activities of different NGOs. Most of these technical staff had a medical background in education with some public health experience or had TB control program experience. In addition, one monitoring officer was appointed for each of the NGO areas. As part of the public-private mix expansion plan, DOTS corners were opened in most of the medical college hospitals. To build and maintain quality services, regular training and refresher courses were conducted for technical staff from the National Tuberculosis Control Program. To engage private practitioners in the program, an area advocacy workshop was arranged in all city corporation areas. Community awareness campaigns were conducted in slums and underserved areas, and were funded by NGO budgets as part of the Global Fund for AIDS, Tuberculosis and Malaria grants. Most of the DOTS centers had been brought under the supervision of the external quality assurance system during 2005 and 2006 to ensure the quality of sputum microscopy. ${ }^{15-17}$

TB cases are suspected and diagnosed according to the national guidelines for TB control. ${ }^{3,18}$ As such, pulmonary TB is suspected in a patient who presents with a persistent cough for three weeks or more, with or without production of sputum, by physicians, public health staff, and/or community health volunteers. Alternatively, extrapulmonary TB cases are typically diagnosed and referred by physicians. Three sputum specimens are examined in patients suspected of having pulmonary TB. New smear-positive pulmonary TB cases are diagnosed when a suspected patient has at least two sputum specimens positive for acid fast bacillus, or with only one sputum specimen positive for acid fast bacillus and chest radiologic abnormalities consistent with active TB or one sputum specimen positive for acid fast bacillus and a culture positive for Mycobacterium tuberculosis. ${ }^{3,18}$ Multidrug resistant TB cases are managed under the multidrug resistance pilot project. $^{2}$ Diagnosed cases are treated with fixed-dose combination therapy under direct supervision (DOTS) for six to eight months. ${ }^{3,18}$ All registered cases are followed to assess improvement at the end of their intensive phase of treatment, as well as upon completion of treatment. ${ }^{3,18}$ Partner NGOs and public-private mix partners send their reports via local government health authorities on a quarterly basis to the National Tuberculosis Control Program (Figure 1). ${ }^{3}$

A total of 20,147 TB patients were registered in city corporation areas during 2006. The case detection rate for new smear-positive TB reached $66.8 \%$ (calculated on an estimated incidence of 103/100,000 population, WHO report), with a sputum conversion rate of $87 \%$ in $2006 .{ }^{19,20}$ The treatment success rate increased from $78 \%$ in 2004 to $84.5 \%$ in $2005 .^{20}$ Trends as of June 2007 are shown in Figure 2 and Table 1. These results suggest that case detection in urban areas has increased considerably compared with the average case detection rate across the entire country (66\% for new smear positive cases in 2006). ${ }^{16}$ This might be due to a nationwide expansion of the TB control program via diverse health care providers. ${ }^{21}$ Similar trends were also reported in other neighboring countries where private health care providers play a significant role. ${ }^{22-24}$ Although cooperation and reporting of new TB cases had increased considerably, treatment adherence to DOTS was still a challenge for the program. 


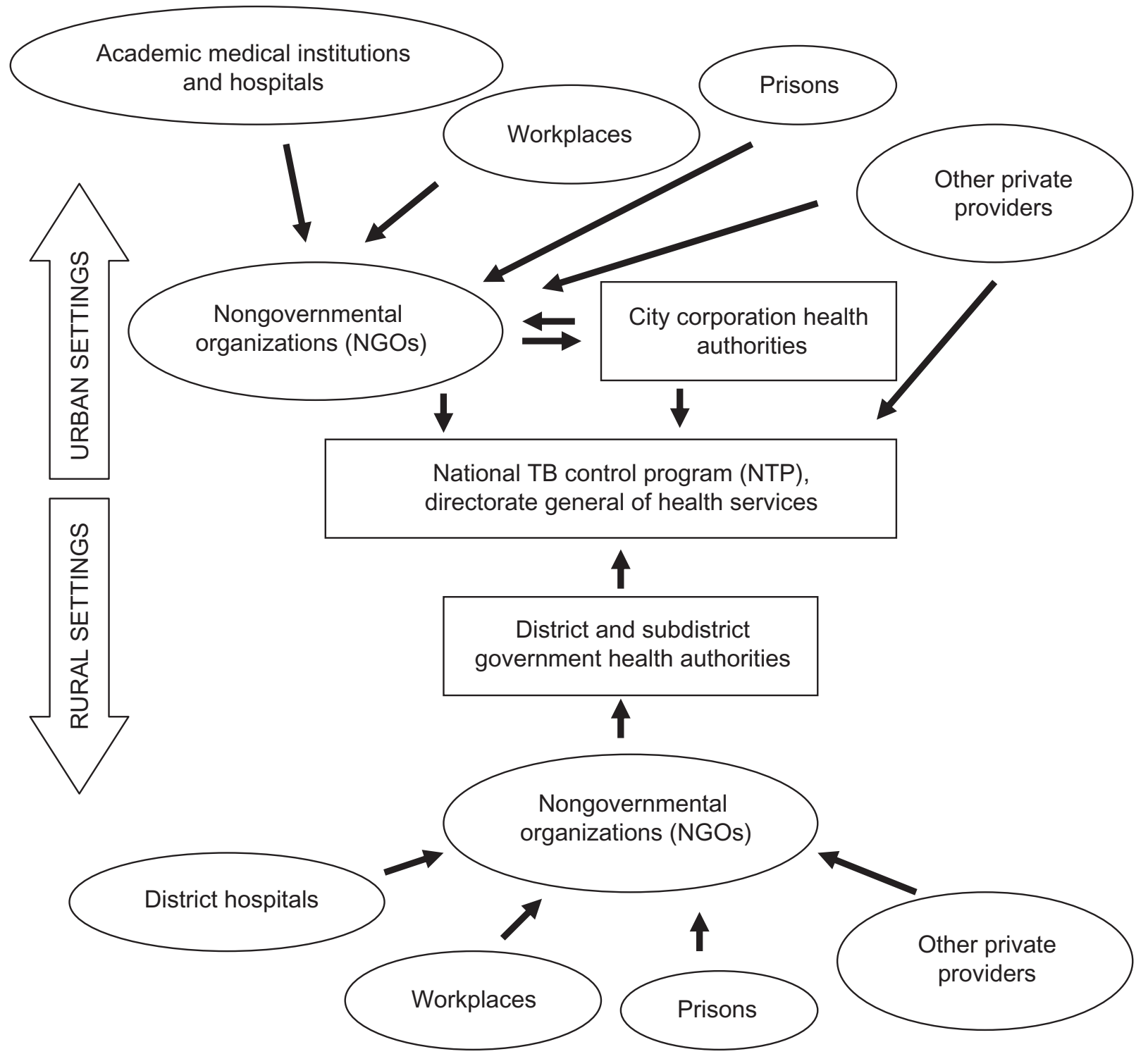

Figure I Schematic diagram for the Management Information System and logistic supply of public private mix DOTS program in Bangladesh.

\section{DOTS in medical academic institutions and hospitals}

DOTS corners were established in 33 medical institutions and government hospitals in 2006. NGOs were given the responsibility to operate these DOTS corners in collaboration with hospital authorities. Advocacy workshops were conducted to inform academics from these institutes on DOTS and national guidelines. A guideline-based referral system was developed to refer patients suspected/diagnosed to have TB, both from inpatient and outpatient departments, to the respective DOTS corner for sputum microscopy. After diagnosis, patients residing in close proximity are registered in the DOTS corners. Others are referred to the center nearest to their residence for registration. Seriously ill inpatients receive initial treatment through attending nurses and, upon discharge, they are referred to their nearest local center.

A total of $3802 \mathrm{~TB}$ patients were registered at 33 hospitals during 2006, compared with 3051 patients at 21 hospitals in 2005 (Table 1). The treatment success rate increased from $76 \%$ in 2004 to $83.2 \%$ in $2005 .{ }^{25}$ Although case notification in hospitals increased in 2006 compared with 2005, treatment success rates remained low compared with the national treatment success rate. ${ }^{6}$ This low treatment success rate might be due to a lack of patient adherence to treatment regimens following hospital discharge. To overcome the challenges 


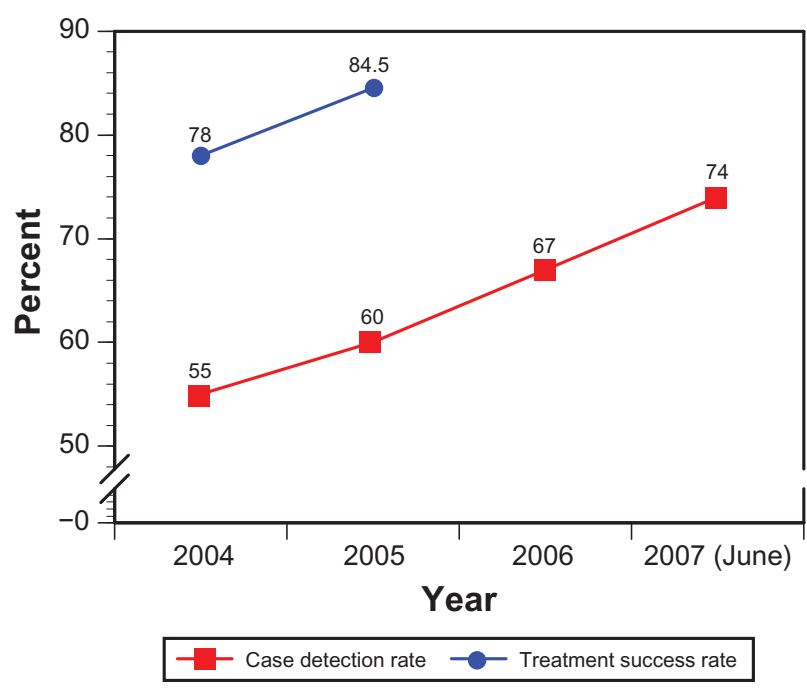

Figure 2 Trends in case detection and treatment success rates in six city corporation areas of Bangladesh.

of low treatment adherence, the referral system needs to be strengthened.

\section{DOTS in workplaces}

DOTS services were implemented in 13 workplaces in Bangladesh, from which the National Tuberculosis Control Program receives individual reports. These workplaces are clustered in the export processing zones, industrial areas, and port areas of Dhaka, Chittagong, Khulna, and Bagerhat. The Dhaka export processing zone is located in the Savar subdistrict, consisting of 94 factories with approximately 72,866 workers (90\% women). ${ }^{26}$ The Chittagong export processing zone consists of 134 industries. Having been a partner of the National Tuberculosis Control Program since 2001, the Youngone Group (Bangladesh) Ltd is one of the largest employers in the Chittagong export processing zone. They have their own health facilities, with a team of 21 doctors, 40 nurses, and 51 health counsellors. ${ }^{26}$

The overcrowded environment in most workplaces could be a potential risk factor for TB infection among workers. Moreover, workers often hide their illness, due to the fear of losing their jobs and being isolated by their coworkers. ${ }^{27,28}$ Regular training on TB control management and sputum microscopy, as well as quarterly monitoring meetings, was conducted for the medical and laboratory staff working in the prescribed areas. In most cases, TB services were integrated within the existing health facilities of the workplaces. Advocacy workshops for factory owners, trade union leaders, supervisors, and workers were conducted to increase awareness for TB suspect identification, referrals, and DOT. DOT was provided by the supervisors, health staff, or coworkers.
Table I Number of tuberculosis cases registered by year

\begin{tabular}{lll}
\hline & $\mathbf{2 0 0 5}$ & $\mathbf{2 0 0 6}$ \\
\hline City corporation area & & \\
New smear-positive cases & 7913 & 9568 \\
Retreatment cases & 1171 & 1297 \\
Smear-negative pulmonary cases & 5320 & 5560 \\
Extrapulmonary cases & 2907 & 3722 \\
Total TB patients & $17,31 \mathrm{I}$ & 20,147 \\
Case detection rate & $60 \%$ & $67 \%$ \\
Medical academic institution hospitals & & \\
New smear-positive cases & 1476 & 1791 \\
Retreatment cases & 421 & 427 \\
Smear-negative pulmonary cases & 650 & 893 \\
Extrapulmonary cases & 504 & 691 \\
Total TB patients & 3051 & 3802 \\
DOTS in workplaces & & \\
New smear-positive cases & 376 & 706 \\
Retreatment cases & 22 & 21 \\
Smear-negative pulmonary cases & 148 & 222 \\
Extrapulmonary cases & 94 & 109 \\
Total TB patients & 640 & 1058 \\
DOTS in prison & & \\
New smear-positive cases & 344 & 268 \\
Retreatment cases & 21 & 30 \\
Smear-negative pulmonary cases & 89 & 59 \\
Extrapulmonary cases & 24 & 44 \\
Total TB patients & 478 & 401 \\
\hline Abbrevations: DOTs, & &
\end{tabular}

Abbreviations: DOTS, directly observed treatment, short-course; TB, tuberculosis.

A total of $1058 \mathrm{~TB}$ patients in 13 workplaces were registered during 2006, compared with 640 patients from 12 workplaces in 2005 . The treatment success rate was 93\% among patients registered in 2005 (details presented in Table 1). ${ }^{29}$ DOT completions were higher among patients in workplaces, possibly due to the fact that these patients become ambulatory within a few weeks after starting their treatment and return to work. Workplace-based treatment adherence was also successful in other reports. ${ }^{6,24,26}$

\section{DOTS in prisons}

Most of the prisons in Bangladesh are filled above capacity. Security measures and administrative formalities make it difficult to provide TB services to the prison communities. Continuation of treatment after prisoners are released is another potential barrier to successful completion of treatment.

According to the public-private mix guidelines, TB services were incorporated into prisons' existing health services, or an NGO was assigned to a prison to implement DOTS. Monthly sputum collection sessions for TB suspects were conducted in the prisons. However, microscopy services were performed in the nearest DOTS microscopy centers. 
DOT services were provided by prison health staff. Following prisoner release, TB cases were transferred to the DOTS center nearest to the prisoner's residence for continuation of treatment.

A total of 401 TB patients were registered in 18 prisons during 2006. The sputum conversion rate increased from $74 \%$ in 2005 to $77.18 \%$ in 2006 . The treatment success rate was $63 \%$ in 2005 . A significant portion of patients were transferred out (18.93\% and 35\%, respectively, for sputum conversion and treatment success). ${ }^{30}$

Some studies have reported higher TB prevalence rates among prisoners compared with the general population. ${ }^{31,32}$ However, existing TB management in the prison health facilities might not be sufficient enough to diagnose and successfully treat TB cases in difficult prison settings. An effective public-private mix DOTS should function to address TB among prisoners.

\section{Conclusion}

It is crucial to engage all care providers with DOTS services in the National Tuberculosis Control Program to cover the entire population and make the services accessible for difficult to reach communities. Successful engagement of academics and clinicians in the DOTS program might eventually facilitate increased case detection and decreased discontinuation of treatment. Quality and performance in areas like prisons and workplaces is still a challenge for the successful implementation of a TB control program.

\section{Acknowledgments}

We gratefully acknowledge the contribution of all the staff of the National Tuberculosis Control Program, World Health Organization Bangladesh, partner NGOs and donor agencies. Urban and public-private mix areas of TB control activities in Bangladesh receive grant support from the Global Fund for AIDS, Tuberculosis and Malaria, the International Union against Tuberculosis and Lung Disease though the Fidelis initiative, and the Research Institute of Tuberculosis, Japan.

\section{Disclosure}

The authors report no conflicts of interest in this work.

\section{References}

1. World Health Organization. Global Tuberculosis Control: Surveillance, Planning and Financing. Geneva, Switzerland: World Health Organization; 2008.

2. World Health Organization. Global Tuberculosis Control: Epidemiology, Strategy, Financing. Geneva, Switzerland: World Health Organization; 2009.
3. National Tuberculosis Control Program. National Guidelines and Operational Manual for Tuberculosis Control, Bangladesh. 3rd ed. Dhaka, Bangladesh: National Tuberculosis Control Program; 2006.

4. Kumaresan JA, Ahsan Ali AK, Parkkali LM. Tuberculosis control in Bangladesh: Success of the DOTS strategy. Int J Tuberc Lung Dis. 1998;2:992-998.

5. Guda DR, Khandaker IU, Parveen SD, Whitson T. Bangladesh: NGO and Public Sector Tuberculosis Service Delivery - Rapid Assessment Results. Bethesda, MD: Quality Assurance Project, University Research Co., LLC, USAID; 2004.

6. National Tuberculosis Control Program. Tuberculosis Control in Bangladesh: Annual Report 2008. Dhaka, Bangladesh: National Tuberculosis Control Programme, Directorate General of Health Services; 2008.

7. National Tuberculosis Control Program. Tuberculosis in Bangladesh: Annual Report. Dhaka, Bangladesh: National Tuberculosis Control Program; 2005.

8. Karim F, Chowdhury AMR, Islam A, Weiss MG. Stigma, gender, and their impact on patients with tuberculosis in rural Bangladesh. Anthropology and Medicine. 2007;14:139-151.

9. Somma D, Thomas BE, Karim F, et al. Gender and socio-cultural determinants of TB-related stigma in Bangladesh, India, Malawi and Colombia. Int J Tuberc Lung Dis. 2008;12:856-866.

10. National Tuberculosis Control Program. The Strategic Plan for TB Control 2006-2010. Dhaka, Bangladesh: National Tuberculosis Control Program; 2005.

11. Ministry of Health. Third Review of the National Tuberculosis Programme in Bangladesh. Dhaka, Bangladesh: Ministry of Health; 2004.

12. Bangladesh Bureau of Statistics. Projected Population Based on Census 2001. Dhaka, Bangladesh: Population Census Wing, Bangladesh Bureau of Statistics; 2007.

13. Khatun J, Roy N. Missed opportunities for reproductive and child health services of clients in urban NGO clinics of Bangladesh. Matern Child Health J. 2006;10:563-570.

14. National Tuberculosis Control Program. Guidelines on Public Private Mix for Tuberculosis Control, Bangladesh. Dhaka, Bangladesh: National Tuberculosis Control Program; 2006.

15. National Tuberculosis Control Program. Laboratory Manual on Smear Microscopy for Tuberculosis and its Quality Control in the NTP of Bangladesh. Dhaka, Bangladesh: National Tuberculosis Control Program; 2004.

16. World Health Organization. Quality Assurance of Sputum Microscopy in DOTS Programmes: Guidelines for Pacific Island Countries. Manila, Philippines: World Health Organization; 2003.

17. Centres for Disease Control and Prevention. External Quality Assessment for AFB Smear Microscopy. Atlanta, GA: Centers for Disease Control and Prevention; 2001.

18. World Health Organization. Treatment of Tuberculosis: Guidelines. 4th ed. Geneva, Switzerland: World Health Organization; 2010.

19. World Health Organization. Global Tuberculosis Control: Surveillance, Planning, Financing. Geneva, Switzerland: World Health Organization; 2006.

20. Khan MH, Islam ABMT, Begum V, et al. TB control in urban Bangladesh: A retrospective analysis. Int J Tuberc Lung Dis. 2007;11 Suppl:274.

21. Rifat M, Rusen ID, Mahmud MH, Nayer I, Islam A, Ahmed F. From mosques to classrooms: Mobilizing the community to enhance case detection of tuberculosis. Am J Public Health. 2008;98: $1550-1552$.

22. Newell JN, Pande SB, Baral SC, Bam DS, Malla P. Control of tuberculosis in an urban setting in Nepal: Public-private partnership. Bull World Health Organ. 2004;82:92-98.

23. Malmborg R, Mann G, Thomson R, Squire SB. Can public-private collaboration promote tuberculosis case detection among the poor and vulnerable? Bull World Health Organ. 2006;84:752-758.

24. World Health Organization. Engaging all Health Care Providers in TB Control: Guidance on Implementing Public-Private Mix Approaches. Geneva, Switzerland: World Health Organization; 2006. 
25. Khan MH, Islam ABMT, Cooreman E, et al. Integration of medical academic institutions and hospitals in TB control programme of Bangladesh. Int J Tuberc Lung Dis. 2007;11 Suppl:274-275.

26. Yesudian HM. Assessment of Tuberculosis Control Activities in Workplaces in Chittagong and Dhaka, Bangladesh. Dhaka, Bangladesh: World Health Organization; 2007.

27. Wagstaff A. Poverty and health sector inequalities. Bull World Health Organ. 2002;80:97-105.

28. Paul-Majumder P, Begum A. The gender imbalances in the export oriented garment industry in Bangladesh. Gender and Development Working Paper 12. Washington, DC: The World Bank Development Research Group; 2000.
29. Khan MH, Islam ABMT, Begum V, et al. Performance of workplace based TB program in Bangladesh. Int J Tuberc Lung Dis. 2007; 11 Suppl:67.

30. Khan MH, Islam ABMT, Begum V, et al. Expansion of DOTS in prisons of Bangladesh. Int J Tuberc Lung Dis. 2007;11 Suppl:280.

31. Banu S, Hossain A, Uddin MKM, et al. Pulmonary tuberculosis and drug resistance in Dhaka Central Jail, the largest prison in Bangladesh. PLoS One. 2010;5:e10759.

32. Rao NA. Prevalence of pulmonary tuberculosis in Karachi central prison. J Pak Med Assoc. 2004;54:413-415.

\section{Publish your work in this journal}

Research and Reports in Tropical Medicine is an international, peerreviewed, open access journal publishing original research, case reports, editorials, reviews and commentaries on all areas of tropical medicine, including: Diseases and medicine in tropical regions; Entomology; Epidemiology; Health economics issues; Infectious disease; Laboratory science and new technology in tropical medicine; Parasitology; Public health medicine/health care policy in tropical regions; and Microbiology. The manuscript management system is completely online and includes a very quick and fair peer-review system. Visit http://www.dovepress. com/testimonials.php to read real quotes from published authors. 